The effect of physical activity on executive function: A brief commentary on definitions, measurement issues, and the current state of the literature

By: Jennifer L. Etnier and Yu-Kai Chang

Etnier, J.L., \& Chang, Y.K. (2009). The effect of physical activity on executive function: A brief commentary on definitions, measurement issues, and the current state of the literature. Journal of Sport and Exercise Psychology, 31, 469-483.

Accepted author manuscript version reprinted, by permission, from Journal of Sport and Exercise Psychology, 2009, 31 (4): 469-483, https://doi.org/10.1123/jsep.31.4.469. (C) Human Kinetics, Inc.

$* * *$ No further reproduction is authorized without written permission from Human
Kinetics, Inc. This version of the document is not the version of record. ***

Abstract:

The purpose of the present commentary is to introduce relevant issues with respect to the measurement of executive function in physical activity studies. Suggested definitions of executive function are introduced, and executive function tasks that are commonly used in the neuropsychological literature are presented and briefly described. The extant literature on physical activity and cognition is discussed, and issues relative to the limitations of this body of literature are raised. In summary, research on the effect of physical activity on executive function is still in its infancy. We encourage researchers in this field to provide a clear definition of executive function, to carefully consider the relevance of published effect sizes to their own research questions, and to consider either providing a logical rationale for their selection of particular executive function measures or to use multiple measures of executive function when exploring relationships between physical activity and executive function.

Keywords: exercise | cognition | neurological assessment

\title{
Article:
}

In her pioneering study (1975), Spirduso reported that active older adults had significantly better cognitive performance than inactive older adults and that their cognitive performance was as good as inactive younger adults. Since the publication of that work, numerous studies have been conducted to examine the relationship between physical activity and cognitive performance. Although the results of empirical studies have not been consistent, authors using meta-analytic techniques and narrative reviews have concluded that a positive relationship exists between physical activity and cognitive performance (Angevaren, Aufdemkampe, Verhaar, Aleman, \& Vanhees, 2008; Colcombe \& Kramer, 2003; Etnier, Nowell, Landers, \& Sibley, 2006; Etnier et al., 1997; Heyn, Abreu, \& Ottenbacher, 2004; Hillman, Erickson, \& Kramer, 2007; Kramer \& Erickson, 2007; Sibley \& Etnier, 2003). 
An important development in this line of research was a recent shift in focus from speed-ofprocessing tasks to executive function tasks. Executive function is a higher order cognitive ability that controls basic, underlying cognitive functions for purposeful, goal-directed behavior and that has been associated with frontal lobe activity. This shift in focus was largely in response to research conducted by Kramer and colleagues. In 1994, based on neuroimaging evidence suggesting that the effects of chronic physical activity were most evident in frontal lobe structure and function, Kramer and his colleagues hypothesized that the effects of physical activity would be most evident for frontally dependent tasks such as executive function tasks (Kramer, Humphrey, Larish, Logan, \& Strayer, 1994). Kramer et al. (1999) then demonstrated empirical support for this hypothesis by showing that chronic physical activity that improved aerobic fitness was particularly beneficial for executive function tasks (i.e., task switching, response compatibility, and stopping). Later, Colcombe and Kramer (2003) provided additional support for this hypothesis through the results of a meta-analytic review of 18 randomized controlled trials (RCTs) of chronic physical activity and cognition conducted with older adults. When statistically summarized, results indicated that chronic physical activity is particularly beneficial for executive function tasks (effect size $=0.68$ ), as compared with controlled tasks (effect size $=$ 0.46 ), spatial tasks (effect size $=0.42$ ), and speeded tasks (effect size $=0.27$ ). These findings have been viewed as especially encouraging given that executive function encompasses many cognitive abilities that have proven essential for daily living (Anderson, Jacobs, \& Anderson, 2008). In addition, Salthouse, Atkinson, and Berish (2003) indicated that some specific executive functions (i.e., inhibition, updating, and time sharing) are potential mediators of age-related cognitive decline in normal adults, supporting the hypothesis that physical activity might serve to delay typical age-related declines in cognition.

However, the focus on executive function in the physical activity and cognition research is a relatively recent development, and researchers interested in studying the relationship between physical activity and executive function might not be aware of the controversy surrounding the term executive function and the relevant measurement issues. Therefore, the purpose of this article is to introduce definitions of executive function and behavioral methods of assessment, to review our current understanding of the relationship between physical activity and executive function, and to introduce relevant considerations for future studies examining this relationship.

\section{Definition of Executive Function}

Executive function, also known as controlled cognition, resource-demanding cognition, or executive control, is generally defined as a "higher level" or "meta-" cognitive function that manages other more basic cognitive functions (Alvarez \& Emory, 2006; Baddeley, 1986; Salthouse, 2007) and the regulation of emotions and attention (Bell \& Deater-Deckard, 2007; Blair \& Diamond, 2008; Lewis et al., 2008) necessary for purposeful and goal-directed behaviors. In the physical activity literature, the focus thus far has been on the cognitive control aspects of executive function, and that is the focus of the discussion to follow. Although the broad definition of executive function as a "central executive" (Baddeley \& Hitch, 1974) is fairly well accepted, there is substantial variety in the description of the specific cognitive functions that underlie executive function (Jurado \& Rosselli, 2007). In attempting to identify and describe these subcomponents of executive function, researchers have relied on conceptual, psychometric, and neuropsychological techniques. 
Kramer et al. (1994) first described executive function in the physical activity literature as consisting of the behaviors of planning, task coordination, initiation and stopping of behaviors, and processing of semantic information. Since that time, the specific descriptors used to describe the subcomponents of executive function have been modified slightly so that executive function in the physical activity literature is now typically described as planning, scheduling, inhibition, and working memory (Colcombe \& Kramer, 2003; Hillman et al., 2006; Kramer et al., 1999). These cognitive functions are consistent with the broad definition of executive function and are congruent with the conceptually based aspects of executive function that have been proposed by others (Anderson, 2002; Banich, 2004; Lezak, Howieson, \& Loring, 2004).

In contrast to providing conceptual definitions of executive function that focus on the particular subcomponents of this construct, other researchers have focused their definition of executive function on the participant's familiarity with the task. For example, Shallice (1990) pointed out that executive functions are not critical for the execution of routine, well-learned behaviors but are specifically activated in novel or unfamiliar circumstances. Hughes and Graham (2002) used the classic distinction between automatic and controlled action and indicated that executive function involves planning and decision making, error correction, the implementation of a novel series of actions, performance in situations that are dangerous or technically difficult, and performance that requires overcoming a robust habitual response. Similarly, Rabbitt (1997) described executive functions as those dealing with novelty, planning and acting on strategies for performance, and using feedback to alter subsequent responses. Thus, these definitions reflect an emphasis on executive function as critical for performance in novel situations or when the performer is required to inhibit a previously learned response.

Other researchers have used psychometric methods to attempt to identify the underlying factors of executive function. Using structural equation modeling, Miyake, Friedman, et al. (2000b) defined executive function as consisting of three basic executive processes: mental set shifting ("shifting"), information updating and monitoring ("updating"), and inhibition of prepotent responses ("inhibition"). Based upon their data, the authors commented that even though the three executive functions are moderately correlated, they are also clearly distinguishable, thus suggesting that these are the three unique subcomponents of executive function.

Another typical approach that has been used to define executive function is based on the performance of frontal lobe-related neuropsychological tasks (Keil \& Kaszniak, 2002; Romine \& Reynolds, 2005). Functioning of the frontal lobe is critical for the performance of complex cognitive functions (Demakis, 2004), and frontal lobe function has been generally linked to executive function. Although the linkage between frontal lobe function and executive function is not a perfect relationship, the terms frontal lobe tasks and executive function tasks are often used interchangeably (Anderson, 1998; Keil \& Kaszniak, 2002). Some researchers (e.g., Cummings, 1993; Duke \& Kaszniak, 2000; Keil \& Kaszniak, 2002) have used evidence from patients with brain damage to the frontal lobe to identify cognitive tasks that are impaired and have inferred that these tasks are related to the construct of executive function. Others have used neuroimaging techniques to attempt to determine the linkage between frontal lobe activity and performance of specific cognitive functions (Carter, Mintun, \& Cohen, 1995; Gilbert \& Burgess, 2008; Taylor, Kornblum, Lauber, Minoshima, \& Koeppe, 1997; Zakzanis, Mraz, \& Graham, 2005). The 
interpretations of executive functions that result from these neuropsychological methods are generally consistent with conceptual and psychometric definitions of executive function.

The premise that executive function describes higher order cognitive functions that are linked to frontal lobe activity is maintained across all of these definitions. However, it is certainly clear that the particular cognitive functions proposed to underlie executive function are not immutable. This is a critical consideration because the definition of executive function and the method for measuring the construct go hand in hand. In fact, Salthouse (2005) argues that executive function is so poorly defined that it is not a construct that can be considered independent from other cognitive abilities. Based upon an analysis of two large data sets, he reported that executive function variables were strongly related to reasoning, perceptual speed abilities, and fluid intelligence (Salthouse, 2005; Salthouse \& Davis, 2006). Evidence of these relationships is logical given that executive function is described as the higher order "executor" that oversees several cognitive functions that represent its subcomponents (Alvarez \& Emory, 2006; Baddeley, 1986; Salthouse, 2007). However, based upon this conclusion, Salthouse (2005) has called for caution in using executive function as though it represents a construct that is distinct from other cognitive dimensions.

The impact of the wide variability in how executive function is defined is important because the particular definition of executive function used to guide the selection of cognitive tasks to assess the construct then influences our ability to interpret the results. Researchers examining the effect of physical activity on executive function are encouraged to consider the complexity of the executive function construct and to give careful consideration to whether to include multiple measures of executive function to identify the effect on the broad construct of executive function or to focus on a specific executive function (e.g., shifting, inhibition, or updating) in their research (Miyake, Emerson, \& Friedman, 2000a; Miyake et al., 2000b; Salthouse, 2007).

\section{Assessment of Executive Function}

The discussion related to the definition of executive function leads directly to a consideration of how to assess executive function. Tasks that are appropriate for the assessment of executive function have typically been identified based either on face validity (Salthouse, 2005) or on the previously described conceptual overlap between executive function and frontal lobe function (Baddeley, Della Salla, Gray, Papagno, \& Spinnler, 1997; Bryan \& Luszcz, 2000; Keil \& Kaszniak, 2002). Given that executive function is an umbrella term used to describe several highlevel cognitive functions that regulate other more basic cognitive processes, its measurement is challenging. In fact, Gilbert and Burgess (2008) point out that even though performance on various measures of executive function tends to be positively correlated, the strength of the relationship is generally low, suggesting, as Miyake et al. (2000b) did, that executive function does not represent a unitary construct.

One of the proposed solutions to the difficulties inherent in trying to find a measure of the complex construct of executive function is to apply multiple assessments that should, as a group, provide a good measure of executive function. Miyake et al. (2000a) suggested that because it is impossible to find a "pure" executive function measure, multiple neuropsychological measures should be used to minimize "task impurity" and to assess the broad construct. Another solution is 
to be clear in understanding the subcomponent(s) that are assessed by a particular behavioral measure (Jurado \& Rosselli, 2007) and to use care in not overgeneralizing to the broader construct of executive function. Regardless of which approach is taken, it is important to be aware of the tasks that have been established as appropriate measures of executive function.

Given the large number of tasks that have been used to assess the executive function construct, it is not our goal to describe all that have been categorized as executive function tasks. Rather, we identify the most commonly reported executive function tasks recognized in eight recent neuropsychological reviews (Alvarez \& Emory, 2006; Bryan \& Luszcz, 2000; Demakis, 2004; Keil \& Kaszniak, 2002; Manchester, Priestley, \& Jackson, 2004; Romine \& Reynolds, 2005; Salthouse et al., 2003; Schillerstrom, Horton, \& Royall, 2005), and we then provide a brief introduction of the most frequently used tasks identified from these reviews. In addition, the tasks identified in these reviews are compared with the top-10 executive function instruments identified by Rabin, Barr and Burton (2005), who conducted a comprehensive assessment of use within the field of clinical neuropsychology.

From the eight published neuropsychological reviews and the top-10 clinical tests of executive function identified by Rabin et al. (2005), 29 tests have been commonly identified as measures of executive function (see Table 1). Of these 29 tests, the Wisconsin Card Sorting Test, the Stroop Test, and the Trail Making Test are most frequently reported as measures of executive function, and, therefore, brief descriptions of these measures are provided.

Table 1. Neuropsychological Tests Proposed to Measure Executive Functions

\begin{tabular}{|c|c|c|c|c|c|c|c|c|c|c|c|}
\hline Study & WCST & $\begin{array}{c}\text { Stroop } \\
\text { Test }\end{array}$ & TMT & VF & TOL & TOH & $\mathbf{C T}$ & DF & FF & COWAT & Other 19 \\
\hline Alvarez \& Emory, 2006 & $*$ & $*$ & & $*$ & & & & & & & \\
\hline Bryan \& Luszcz, 2000 & $*$ & $*$ & & $*$ & $*$ & & & & & $*$ & $*$ \\
\hline Demakis, 2004 & & $*$ & $*$ & & & & $*$ & & & & \\
\hline Keil \& Kaszniak, 2002 & $*$ & $*$ & $*$ & & $*$ & $*$ & $*$ & $*$ & $*$ & & $*$ \\
\hline Manchester et al., 2004 & $*$ & $*$ & $*$ & & & & $*$ & & & $*$ & $*$ \\
\hline Rabin, Barr, \& Burton, 2005 & $*$ & $*$ & $*$ & & $*$ & & $*$ & & $*$ & $*$ & $*$ \\
\hline Romine \& Reynolds, 2005 & $*$ & & & $*$ & $*$ & $*$ & & $*$ & & & $*$ \\
\hline Salthouse et al., 2003 & $*$ & & $*$ & $*$ & $*$ & $*$ & & & $*$ & & \\
\hline Schillerstrom et al., 2005 & $*$ & $*$ & $*$ & $*$ & & $*$ & & & & & $*$ \\
\hline
\end{tabular}

Note. $*$ Recognized as a measure of executive function; WCST $=$ Wisconsin Card Sorting Test, TMT $=$ Trial Making

Test, $\mathrm{VF}=$ Verbal Fluency, $\mathrm{TOL}=$ Tower of London Test, $\mathrm{TOH}=$ Tower of Hanoi, $\mathrm{CT}=$ Category Test, $\mathrm{DF}=$

Design Fluency, FF = Figure Fluency, COWAT $=$ Controlled Oral Word Association Test, Other $19=$ Other tasks related to executive function: Cognitive Estimated Test, Self-Ordered Pointing Task, Neuropsychological Tower, Clock Drawing Test, Objects Test, Graphic Pattern Generation, Sequence Generation Test, Multiple Errands Test, Porteus Mazes, Rey-Osterrieth Complex Figure, Raven's Colored Progressive Matrices, Tactual Performance Test, Tinkertoy Test, Virtual Planning Test, Visual Search Test, WAIS-R/WAIS-III, WAIS-R/WAIS-III/WASI Block Design, WAIS-R/WAIS-III Picture Arrangement, Wheelbarrow Assembly.

\section{Wisconsin Card Sorting Test}

The Wisconsin Card Sorting Test (WCST), which has been reported to be commonly used by over $75 \%$ of neuropsychologists in clinical settings, is the most popular test of executive function (Delis, Kaplan, \& Kramer, 2001; Greve, Stickle, Love, Bianchini, \& Stanford, 2005; Rabin et al., 2005). The WCST requires participants to sort cards based upon one of the three characteristics 
of the card (color, form, number) and using feedback from the examiner to identify the appropriate sorting characteristic, which changes after every 10 cards. Performance on this task requires that the participant identifies the characteristic of the card that is being used for sorting, maintains the task set until feedback demands otherwise, avoids the tendency to use an incorrect sorting characteristic, and inhibits the previous response when it is no longer appropriate (Salthouse et al., 2003). Performance on the WCST is sensitive to frontal lobe damage and is purported to assess the executive functions of switching, inhibition, updating, and selective attention (Alvarez \& Emory, 2006; Greve et al., 2005).

\section{Stroop Test}

The Stoop Test (Stroop, 1935), also referred to as the color-naming task, is another frequently used measure of executive function. In this task, participants are required to identify the color of the stimulus in each of three conditions. In the word condition, also known as the neutral condition, the stimulus is an irrelevant symbol string printed in colored ink, such as XXXX or OOOO printed in red ink, or a color patch such as a red square. In the color condition, also known as the congruent condition, the stimulus is a color name that matches the ink color in which it is written, such as RED printed in red ink. In the color-word condition, also known as the interference or incongruent condition, the stimulus is a color name printed in a different ink color, such as BLUE printed in red ink. In the examples provided here, the correct answer is red for all conditions. In all three conditions, participants respond to a fixed number of stimuli and the time to complete the task is used as the measure of performance.

Performance on the word and color conditions has typically been used as a measure of speed of processing. On the other hand, performance on the color-word condition has been used as a measure of executive function. Several different scoring methods have been used for the colorword condition, including simply using the time to completion of the color-word condition, calculating the difference between time for the color-word condition and time for either the color or the word condition, or calculating the difference between time for the color-word condition and the average time for the color and word conditions. The Stroop Test is described as assessing the ability to inhibit a habitual response, although selective attention and shifting ability might also be involved (Miyake et al., 2000b; Pachana, Thompson, Marcopulos, \& Yoash-Gantz, 2004).

\section{Trail Making Test}

The Trail Making Test (TMT) is a timed paper-and-pencil task that consists of two separate parts. Part A (TMT-A) involves drawing a line to connect consecutive numbers (i.e., from 1 to 25). Part B (TMT-B) requires the participant to connect numbers and letters in an alternating progressive sequence (i.e., 1 to A to 2 to B). To perform the TMT successfully requires a variety of abilities, including number recognition, visual scanning with a motor component, and mental flexibility (Corrigan \& Hinkeldey, 1987). In addition, performance on the TMT-B has increased requirements (as compared with the TMT-A) in terms of task-set inhibition ability, cognitive flexibility, and the ability to maintain a response set (Arbuthnott \& Frank, 2000; Kortte, Horner, $\&$ Windhan, 2002). To isolate the executive function requirements of the TMT-B from the general processes of perceiving and responding, the difference between TMT-B and TMT-A or 
the ratio of TMT-B to TMT-A has typically been used as the measure of executive function (Arbuthnott \& Frank, 2000; Salthouse et al., 2003).

\section{Current Evidence Regarding the Effects of Physical Activity on Executive Function}

Given the challenges in defining executive function and the large number of tests that have been identified as measures of executive function, it is perhaps not surprising that the early work in the area of physical activity and executive function was designed merely to establish a basic understanding of this relationship. We believe our field is now ready to further advance our understanding by addressing some of the subtleties of the relationship through a more explicit consideration of the complex construct of executive function and of the current state of the literature. We reviewed the extant literature on physical activity and cognitive performance to help provide direction for future research.

First, we examined the cognitive measures used in empirical studies included in two metaanalyses on physical activity and cognitive function (Etnier et al., 1997, 2006). The cognitive measures used in these studies were categorized as being executive function tasks (if they were included in Table 1) or as nonexecutive function tasks. Although not an exhaustive search, an examination of the 152 studies identified in this fashion is informative with respect to understanding the current status of the research on physical activity and executive function.

Of interest is the fact that relatively few studies report on the effects of physical activity on tasks identified by neuropsychologists as measures of executive function. Of the over 128 different cognitive tests used in the empirical studies included in these meta-analyses, only 10 of these are tests that have been identified as commonly used measures of executive function (listed in Table 1). This is likely to be indicative of the relatively recent interest in executive function in exercise psychology and is illustrative of how little data we actually have available on the effects of physical activity on executive function. Another way of looking at this is that only 10 of the 29 executive function tasks identified as being most commonly used in the neuropsychology literature have been used in studies testing the relationship between physical activity and cognitive performance. The lack of overlap between measurements used in the neuropsychological field and in the physical activity literature may be indicative of a lack of consistency in terms of how executive function has been operationalized in the two fields.

As a second step in reviewing the extant literature, we considered the two meta-analytic reviews of the literature on physical activity and cognitive performance that were focused specifically on executive function. Colcombe and Kramer (2003) meta-analytically reviewed 18 RCTs that were published between 1966 and 2001 and that tested the effects of chronic physical activity on cognitive performance by older adults (55-81 years). Angevaren et al. (2008) statistically analyzed the results of 11 RCTs of physical activity on cognitive function that were available in 2005 and that focused on cognitively normal adults over 55 years of age. Two comments with respect to these reviews are of note. First, the overall average effect sizes for executive function tasks reported by Colcombe and Kramer and by Angevaren et al. are dramatically different. Angevaren et al. reported a small positive effect (effect size $=0.23$ ) whereas Colcombe and Kramer reported a moderate effect (effect size $=0.68$ ). The difference in effect size might be due to a number of factors (or a combination thereof). One possible reason for the differences is that 
Colcombe and Kramer included older adults with cognitive impairment in their review whereas Angevaren et al. included only studies with healthy older adults. This explanation for the differing effect sizes is consistent with the fact that Heyn et al. (2004) reported an effect size of 0.57 for randomized trials testing the effects of physical activity on cognitive performance by older ( $>65$ years) impaired adults. A second explanation for the disparate findings is that the authors used slightly different inclusion criteria resulting in only five studies appearing in both meta-analyses. If the variability in the overall effect sizes from these two meta-analyses is due to the different samples of studies included, this clearly implies that neither effect size should be considered a perfectly reliable indicant of the population effect size. A third possible reason for the discrepant effect sizes may be related to the particular method of coding studies as testing executive function. Angevaren et al. identified tasks as measures of executive function based on existing neuropsychological reviews (Kessels, Aleman, Verhagen, \& Luijtelaar, 2000; Lezak et al., 2004). Colcombe and Kramer did not reference their method for identifying studies as measures of executive function and included tasks such as the dementia scales, sequence learning, supraverbal span, and remote memory, which have not consistently been identified as executive function tasks in the neuropsychological field. Regardless of the explanation for the differing results, it is important to recognize that the moderate and promising effect size reported by Colcombe and Kramer for executive function was not observed in a more recent metaanalysis of RCTs with older adults and, therefore, might not provide a robust estimate of the effect that can be attained in a given empirical study.

A second comment with respect to these meta-analyses is that it is important to recognize that both of these meta-analytic reviews (Angevaren et al., 2008; Colcombe \& Kramer, 2003) were focused on studies implementing chronic physical activity interventions with older adults. Thus, researchers should recognize that these effect sizes are not appropriate to support power analyses for studies with other populations or for studies assessing the effects of acute exercise on executive function.

Another relevant comment that is apparent from reviewing the extant literature is that only a handful of studies have examined executive function using multiple measures (e.g., Davis et al., 2007; Dietrich \& Sparling, 2004; Kramer et al., 1999), whereas most physical activity studies have included only one or two measures of executive function (e.g., Bixby et al., 2007; Colcombe et al., 2004; Coles \& Tomporowski, 2008; Erickson et al., 2007; Hillman, Castelli, \& Buck, 2005; Hillman, Snook, \& Jerome, 2003; Hogervorst, Riedel, Jeukendrup, \& Jolles, 1996; Kamijo et al., 2004a; Kamijo et al., 2004b; Sibley \& Beilock, 2007). Thus, the findings from these studies provide an indication of the relationship between physical activity and specific executive functions but obviously do not provide insights about aspects of executive function not assessed and may not include enough representative measures of executive function to appropriately represent the broader construct. In addition, as previously mentioned, most of the measures that have been used in the physical activity literature are not as commonly used in the neuropsychological literature, limiting our ability to compare across these fields. Lastly, when studies have used multiple measures of executive function, physical activity researchers have typically not pursued an understanding of reasons for potential task specificity in the results. This is understandable given the relative infancy of this area of research; however, future research is clearly necessary to enhance our understanding of which executive functions are most affected by physical activity. 
As a final comment on the existing research, there are a number of recent empirical studies in which the flankers test or a go/no go paradigm has been used to assess executive function (Colcombe et al., 2003; Colcombe et al., 2006; Colcombe et al., 2004; Erickson et al., 2007; Hillman, Belopolsky, Snook, Kramer, \& McAuley, 2004; Hillman et al., 2006; Hillman et al., 2003; Kamijo et al., 2004a; Kamijo et al., 2004b; Kramer \& Hillman, 2006). The reason for choosing these particular measures of executive function is likely related to their compatibility with the use of neuroimaging or electroencephalographic techniques. These studies have allowed for an examination of the potential underlying mechanisms of frontal lobe function in furthering our understanding of the physical activity and executive function relationship. However, these tasks are not used frequently in the neuropsychology literature. Although the use of a variety of tasks to assess particular aspects of executive function (e.g., inhibition) may provide converging evidence regarding the effects of physical activity, the use of tasks that are not popular in the field of neuropsychology might also increase the challenges that face us in furthering our understanding of the relationship between physical activity and executive function because the findings from these studies are not readily comparable to the extant neuropsychological literature. Thus, researchers interested in using psychophysiological techniques to understand executive functions might consider also using tasks, such as the Stroop task, that are amenable to the use of these techniques (Langenecker, Nielson, \& Rao, 2004) and that are more commonly used in neuropsychology.

\section{Conclusion}

Research examining the effect of physical activity on executive function is still in its infancy and several issues are worth consideration as we add to our understanding of this relationship. First and foremost, it is critical that researchers in this area establish a clear rationale and justification for their decision to focus on executive function as their cognitive outcome of interest. Although the moderate effect size reported for executive function tasks in the Colcombe and Kramer (2003) study is frequently used to justify a focus on executive function tasks, we believe it may be premature for researchers interested in the effects of physical activity on cognition to focus exclusively on executive function tasks particularly in cases in which their study design is dissimilar to that of the studies included in that meta-analysis. For example, researchers interested in the effects of acute exercise on cognition or on the benefits of physical activity for cognitive performance by children, young or middle aged adults, or older adults without cognitive impairment might not observe the same moderate effects as reported by Colcombe and Kramer (2003) for older adults participating in chronic exercise interventions.

Second, given the breadth of the executive function construct, it is important for researchers in the area of physical activity and executive function to fully appreciate the impact that their choice of executive function measures can have on the outcome of their research. In the extant literature on physical activity and cognition, there are only a handful of studies that have used multiple of measures of executive function and there are no studies of which we are aware in which executive function has been assessed comprehensively. In future research, we encourage researchers to base their selection of executive function tasks on a theoretical framework and on empirical evidence supporting the sensitivity of the task to the particular physical activity effects of interest in the population of interest. In addition, it is recommended that researchers consider 
using multiple measures of executive function to assess the construct more broadly, to minimize the effects of task impurity on the outcomes (Miyake et al., 2000a), and to advance our understanding of the task specificity of the effects.

Third, there is evidence indicating that executive function develops with increasing age in children (Anderson, 2002), suggesting that physical activity might be particularly beneficial for this age group. Although limited, there is evidence that fitness level is positively associated with executive function in children (Buck, Hillman, \& Castelli, 2008; Hillman et al., 2005) and that children who participate in a 15 -week physical activity program experience significant improvements in executive function (Davis et al., 2007). These promising results suggest that further empirical research is warranted with this population.

The finding that participation in physical activity can serve to benefit cognitive functioning (Angevaren et al., 2008; Colcombe \& Kramer, 2003; Etnier et al., 2006; Etnier et al., 1997; Heyn et al., 2004; Sibley \& Etnier, 2003) is one that has garnered excitement in scientists, practitioners, and the general public. However, the current literature with regard to the benefits for executive function is somewhat limited in scope. As we continue to develop this body of literature, it is important that we pay attention to the extant literature in exercise psychology, neuropsychology, and other related fields so that we are specific in our definitions, exact in our operationalizing of cognitive constructs, and justified in our focus on specific types of executive function.

\section{References}

Alvarez, J.A., \& Emory, E. (2006). Executive function and the frontal lobes: A meta-analytic review. Neuropsychology Review, 16(1), 17-42.

Anderson, P. (2002). Assessment and development of executive function (EF) during childhood. Child Neuropsychology, 8(2), 71-82.

Anderson, V. (1998). Assessing executive functions in children: Biological, psychological, and developmental considerations. Neuropsychological Rehabilitation, 8(3), 319-349.

Anderson, V., Jacobs, R., \& Anderson, P. (2008). Executive functions and the frontal lobes: A lifespan perspective. Philadelphia: Psychology Press.

Angevaren, M., Aufdemkampe, G., Verhaar, H.J.J., Aleman, A., \& Vanhees, L. (2008). Physical activity and enhanced fitness to improve cognitive function in older people without known cognitive impairment. Cochrane Database of Systematic Reviews (Online : Update Software), 3, $1-96$.

Arbuthnott, K., \& Frank, J. (2000). Trail making test, part B as a measure of executive control: Validation using a set-switching paradigm. Journal of Clinical and Experimental Neuropsychology, 22(4), 518-528.

Baddeley, A.D. (1986). Working memory. Oxford: Clarendon Press. 
Baddeley, A.D., Della Salla, S., Gray, C., Papagno, C., \& Spinnler, H. (1997). Testing central executive function testing with a pencil-and-paper test. In P. Rabbitt (Ed.), Methodology of frontal and executive function (pp. 61-80). Hove, UK: Psychology Press.

Baddeley, A.D., \& Hitch, G. (1974). Working memory. In The psychology of learning and motivation: Advances in research and theory (Vol. 8, pp. 47-89). New York: Academic Press.

Banich, M.T. (2004). Cognitive neuroscience and neuropsychology. Boston: Houghton Mifflin.

Bell, M.A., \& Deater-Deckard, K. (2007). Biological systems and the development of selfregulation: Integrating behavior, genetics, and psychophysiology. Journal of Developmental and Behavioral Pediatrics, 28(5), 409-420.

Bixby, W.R., Spalding, T.W., Haufler, A.J., Deeny, S.P., Mahlow, P.T., Zimmerman, J., et al. (2007). The unique relation of physical activity to executive function in older men and women. Medicine and Science in Sports and Exercise, 39(8), 1408-1416.

Blair, C., \& Diamond, A. (2008). Biological processes in prevention and intervention: The promotion of self-regulation as a means of preventing school failure. Development and Psychopathology, 20(3), 899-911.

Bryan, J., \& Luszcz, M.A. (2000). Measurement of executive function: Considerations for detecting adult age differences. Journal of Clinical and Experimental Neuropsychology, 22(1), 40-55.

Buck, S.M., Hillman, C.H., \& Castelli, D.M. (2008). The relation of aerobic fitness to stroop task performance in preadolescent children. Medicine and Science in Sports and Exercise, 40(1), $166-172$.

Carter, C.S., Mintun, M., \& Cohen, J.D. (1995). Interference and facilitation effects during selective attention: An H215O PET study of Stroop task performance. NeuroImage, 2, 264-272.

Colcombe, S.J., Erickson, K.I., Raz, N., Webb, A.G., Cohen, N.J., McAuley, E., et al. (2003). Aerobic fitness reduces brain tissue loss in aging humans. Journal of Gerontology: Biological Sciences and Medical Sciences, 58A(2), 176-180.

Colcombe, S.J., Erickson, K.I., Scalf, P.E., Kim, J.S., Prakash, R., McAuley, E., et al. (2006). Aerobic exercise training increases brain volume in aging humans. Journal of Gerontology: Biological Sciences and Medical Sciences, 61A(11), 1166-1170.

Colcombe, S.J., \& Kramer, A.F. (2003). Fitness effects on the cognitive function of older adults: A meta-analytic study. Psychological Science, 14(2), 125-130. 
Colcombe, S.J., Kramer, A.F., Erickson, K.I., Scalf, P.E., McAuley, E., Cohen, N.J., et al. (2004). Cardiovascular fitness, cortical plasticity, and aging. Proceedings of the National Academy of Sciences of the United States of America, 101(9), 3316-3321.

Coles, K., \& Tomporowski, P.D. (2008). Effects of acute exercise on executive processing, short-term and long-term memory. Journal of Sports Sciences, 26(3), 333-344.

Corrigan, J.D., \& Hinkeldey, N.S. (1987). Relationships between parts A and B of the Trail Making Test. Journal of Clinical Psychology, 43(4), 402-409.

Cummings, J.L. (1993). Frontal-subcortical circuits and human behavior. Archives of Neurology, 50, 873-880.

Davis, C.L., Tomporowski, P.D., Boyle, C.A., Waller, J.L., Miller, P.H., Naglieri, J.A., et al. (2007). Effects of aerobic exercise on overweight children's cognitive functioning: A randomized controlled trial. Research Quarterly for Exercise and Sport, 78(5), 510-519.

Delis, D.C., Kaplan, E., \& Kramer, J.H. (2001). Delis-Kaplan executive function system. New York: Psychological Corporation.

Demakis, G.J. (2004). Frontal lobe damage and tests of executive processing: A meta-analysis of the category test, stroop test, and trail-making test. Journal of Clinical and Experimental Neuropsychology, 26(3), 441-450.

Dietrich, A., \& Sparling, P.B. (2004). Endurance exercise selectively impairs prefrontaldependent cognition. Brain and Cognition, 55, 516-524.

Duke, L.M., \& Kaszniak, A.W. (2000). Executive functions in degenerative dementias: A comparative review. Neuropsychology Review, 10, 75-99.

Erickson, K.I., Colcombe, S.J., Elavsky, S., McAuley, E., Korol, D.L., Scalf, P.E., et al. (2007). Interactive effects of fitness and hormone treatment on brain health in postmenopausal women. Neurobiology of Aging, 28, 179-185.

Etnier, J.L., Nowell, P.M., Landers, D.M., \& Sibley, B.A. (2006). A meta-regression to examine the relationship between aerobic fitness and cognitive performance. Brain Research Reviews, 52, 119-130.

Etnier, J.L., Salazar, W., Landers, D.M., Petruzzello, S.J., Han, M., \& Nowell, P. (1997). The influence of physical fitness and exercise upon cognitive functioning: A meta-analysis. Journal of Sport \& Exercise Psychology, 19, 249-277.

Gilbert, S.J., \& Burgess, P.W. (2008). Executive function. Currents in Modern Biology, 18(3), R110-R114. 
Greve, K.W., Stickle, T.R., Love, J.M., Bianchini, K.J., \& Stanford, M.S. (2005). Latent structure of the Wisconsin Card Sorting Test: A confirmatory factor analytic study. Archives of Clinical Neuropsychology, 20, 355-364.

Heyn, P., Abreu, B.C., \& Ottenbacher, K.J. (2004). The effects of exercise training on elderly persons with cognitive impairment and dementia: A meta-analysis. Archives of Physical Medicine and Rehabilitation, 85, 1694-1704.

Hillman, C.H., Belopolsky, A., Snook, E.M., Kramer, A.F., \& McAuley, E. (2004). Physical activity and executive control: Implications for increased cognitive health during older adulthood. Research Quarterly for Exercise and Sport, 75, 176-185.

Hillman, C.H., Castelli, D.M., \& Buck, S.M. (2005). Aerobic fitness and neurocognitive function in healthy preadolescent children. Medicine and Science in Sports and Exercise, 37(11), 19671974.

Hillman, C.H., Erickson, K.I., \& Kramer, A.F. (2008). Be smart, exercise your heart: Exercise effects on brain and cognition. Nature Reviews. Neuroscience, 9, 58-65.

Hillman, C.H., Motl, R.W., Pontifex, M.B., Posthuma, D., Stubbe, J.H., Boomsma, D.I., et al. (2006). Physical activity and cognitive function in a cross-section of younger and older community-dwelling individuals. Health Psychology, 25(6), 678-687.

Hillman, C.H., Snook, E.M., \& Jerome, G.J. (2003). Acute cardiovascular exercise and executive control function. International Journal of Psychophysiology, 48, 307-314.

Hogervorst, E., Riedel, W., Jeukendrup, A., \& Jolles, J. (1996). Cognitive performance after strenuous physical exercise. Perceptual and Motor Skills, 83, 479-488.

Hughes, C., \& Graham, A. (2002). Measuring executive functions in childhood: Problems and solutions? Child and Adolescent Mental Health, 7(3), 131-142.

Jurado, M.B., \& Rosselli, M. (2007). The elusive nature of executive functions: A review of our current understanding. Neuropsychology Review, 17(3), 213-233.

Kamijo, K., Nishihira, Y., Hatta, A., Kaneda, T., Kida, T., Higashiura, T., et al. (2004a). Changes in arousal level by differential exercise intensity. Clinical Neurophysiology, 115, 26932698.

Kamijo, K., Nishihira, Y., Hatta, A., Kaneda, T., Wasaka, T., Kida, T., et al. (2004b). Differential influences of exercise intensity on information processing in the central nervous system. European Journal of Applied Physiology, 92, 305-311.

Keil, K., \& Kaszniak, A.W. (2002). Examining executive function in individuals with brain injury: A review. Aphasiology, 16(3), 305-335. 
Kessels, R.P.C., Aleman, A., Verhagen, W.I.M., \& Luijtelaar, E.L.J.M. (2000). Cognitive functioning after whiplash injury: A meta-analysis. Journal of the International Neuropsychological Society, 6, 271-278.

Kortte, K.B., Horner, M.D., \& Windhan, W.K. (2002). The Trail Making Test, part B: Cognitive flexibility or ability to maintain set? Applied Neuropsychology, 9(2), 106-109.

Kramer, A.F., \& Erickson, K.I. (2007). Capitalizing on cortical plasticity: Influence of physical activity on cognition and brain function. Trends in Cognitive Sciences, 11(8), 342-348.

Kramer, A.F., Hahn, S., Cohen, N.J., Banich, M.T., McAuley, E., Harrison, C.R., et al. (1999). Ageing, fitness and neurocognitive function. Nature, 400, 418-419.

Kramer, A.F., \& Hillman, C.H. (2006). Aging, physical activity, and neurocognitive function. In E.O. Acevedo \& P. Ekkekakis (Eds.), Psychobiology of physical activity (pp. 45-60).

Champaign, IL: Human Kinetics.

Kramer, A.F., Humphrey, D.G., Larish, J.F., Logan, G.D., \& Strayer, D.L. (1994). Aging and inhibition: Beyond a unitary view of inhibitory processing in attention. Psychology and Aging, 9(4), 491-512.

Langenecker, S.A., Nielson, K.A., \& Rao, S.M. (2004). fMRI of healthy older adults during Stroop interference. NeuroImage, 21, 192-200.

Lewis, M.D., Granic, I., Lamm, C., Zelazo, P.D., Stieben, J., Todd, R.M., et al. (2008). Changes in the neural bases of emotion regulation associated with clinical improvement in children with behavior problems. Development and Psychopathology, 20(3), 913-939.

Lezak, M.D., Howieson, D.B., \& Loring, D.W. (2004). Neuropsychological assessment. New York: Oxford University Press.

Manchester, D., Priestley, N., \& Jackson, H. (2004). The assessment of executive functions: Coming out of the office. Brain Injury : [BI], 18(11), 1067-1081.

Miyake, A., Emerson, M.J., \& Friedman, M.A. (2000a). Assessment of executive functions in clinical settings: Problems and recommendations. Seminars in Speech and Language, 21(2), 169-183.

Miyake, A., Friedman, N.P., Emerson, M.J., Witzki, A.H., Howerter, A., \& Wager, T.D. (2000b). The unity and diversity of executive functions and their contributions to complex "frontal lobe" tasks: A latent variable analysis. Cognitive Psychology, 41, 49-100. 
Pachana, N.A., Thompson, L.W., Marcopulos, B.A., \& Yoash-Gantz, R. (2004). California Older Adult Stroop Test (COAST): Development of a Stroop test adapted for geriatric populations. Clinical Gerontologist, 27(3), 3-22.

Rabbitt, P. (1997). Introduction: Methodologies and models in the study of executive function. In P. Rabbitt (Ed.), Methodology of frontal and executive function (pp. 1-38). UK: Psychology Press.

Rabin, L.A., Barr, W.B., \& Burton, L.A. (2005). Assessment practices of clinical neuropsychologists in the United States and Canada: A survey of INS, NAN, and APA Division 40 members. Archives of Clinical Neuropsychology, 20, 33-65.

Romine, C.B., \& Reynolds, C.R. (2005). A model of the development of frontal lobe functioning: Findings from a meta-analysis. Applied Neuropsychology, 12(4), 190-201.

Salthouse, T.A. (2005). Relations between cognitive abilities and measures of executive functioning. Neuropsychology, 19, 532-545.

Salthouse, T.A. (2007). Exercise and mental resources: Methodological problems. In W.W. Spirduso, L.W. Poon, \& W. Chodzko-Zajko (Eds.), Exercise and its mediating effects on cognition (Vol. 2, pp. 111-118). Champaign, IL: Human Kinetics.

Salthouse, T. A., Atkinson, T.M., \& Berish, D.E. (2003). Executive functioning as a potential mediator of age-related cognitive decline in normal adults. Journal of Experimental Psychology. General, 132(4), 566-594.

Salthouse, T.A., \& Davis, H.P. (2006). Organization of cognitive abilities and neuropsychological variables across the lifespan. Developmental Review, 26, 31-54.

Schillerstrom, J.E., Horton, M.S., \& Royall, D.R. (2005). The impact of medical illness on executive function. Psychosomatics, 46, 508-516.

Shallice, T. (1990). From neuropsychology to mental structure. New York: Cambridge University Press.

Sibley, B.A., \& Beilock, S.L. (2007). Exercise and working memory: An individual differences investigation. Journal of Sport \& Exercise Psychology, 29, 783-791.

Sibley, B.A., \& Etnier, J.L. (2003). The relationship between physical activity and cognition in children: A meta-analysis. Pediatric Exercise Science, 15(3), 243-256.

Spirduso, W.W. (1975). Reaction and movement time as a function of age and physical activity level. Journal of Gerontology, 30, 435-440. 
Stroop, J.R. (1935). Studies of interference in serial verbal reactions. Journal of Experimental Psychology, 18, 643-662.

Taylor, S.F., Kornblum, S., Lauber, E.J., Minoshima, S., \& Koeppe, R.A. (1997). Isolation of specific interference processing in the Stroop task: PET activation studies. NeuroImage, 6, 8192.

Zakzanis, K.K., Mraz, R., \& Graham, S.J. (2005). An fMRI study of the Trail Making Test. Neuropsychologia, 43(13), 1878-1886. 